\title{
Communication via Intermediaries
}

\author{
Maksymilian Kwiek*† \\ University of Southampton
}

January 12, 2020

\begin{abstract}
A model of communication with two features is presented: inattentive receivers, who are heterogeneous in their inattention, and the intermediaries, whose preferences are not aligned with each other or with the preferences of the initial sender. The sender faces a dilemma how to shape her message to maneuver the intermediaries into telling the final receivers what she considers important. A sincere communication policy and competition between the intermediaries are optimal if three conditions hold: the intermediaries have opposite preferences as to what the final receivers should learn, they are symmetric in their communication capacities and the sender is benevolent towards final receivers. Otherwise, the sender may benefit from a more handson intervention - selecting a self-censoring communication policy over sincerity or a single intermediary over competition.

Keywords: inattention; communication; information theory.

JEL Code: D83
\end{abstract}

*m.kwiek@soton.ac.uk. If you want to send comments to me anonymously, please use this form: goo.gl/JS9D5j

${ }^{\dagger}$ I thank Jakub Steiner and the participants of 2016 CRETA Conference at the University of Warwick for helpful comments. I would like to thank Wioletta Dziuda, Ying Chen, Thomas Gall, and other participants of the Workshop at the University of Southampton and $\mathrm{Ce}^{2}$ Workshop 2014 in Warsaw, for many insightful comments on an earlier version of this paper. 


\section{Introduction}

Consider an expert communicating to a public via specialized intermediaries such as news reporters. This paper investigates policies that the expert may apply in an effort to manipulate the reporters into telling a story that is the closest to what she prefers.

The main premise is that the listeners are not ready or capable to listen to the messages beyond a certain point. I will say that the listeners are inattentive. Communication is fully flexible within this constraint. The dilemma of the expert (henceforth, the Sender) comes from the fact that she loses the ownership of the message the moment the reporters (henceforth, the Intermediaries) hear it. For this reason, she has to be careful about what she says and to whom.

The "what" question leads to the following trade-off. The Sender may inform the Intermediary sincerely about the issues she considers important, thus providing information of good quality to the relatively attentive agents, but at a price of the Intermediary skewing information designated for the less attentive receivers. Alternatively, the Sender may apply a policy of selfcensorship, whereby she refuses to share information that the Intermediary would like to over-communicate. The inattentive segment of the public is better informed, but the message for the more attentive segment is not as good as in the sincere policy. One can interpret self-censorship as a populist policy targeting inattentive part of the public at the expense of the more attentive elites.

The "to whom" question goes beyond a simple decision as to which Intermediary should be selected; it pertains to the potential benefits of competition. Competition among the Intermediaries can be induced by transparency, understood here as revealing information to all Intermediaries, for example, in a press conference. Competition may also be prevented by using an exclusive interview with a single Intermediary.

The main results can be summarized as follows. The Sender will optimally use sincere policy and transparency if three conditions hold. Firstly, the Intermediaries have opposite preferences as to what the final Receivers should know; secondly, they are symmetric in their communication capacities; finally, the Sender is benevolent in the sense of having the same preferences as the final receivers. Essentially, competition disciplines the Intermediaries towards what the final Receiver (and the Sender) prefers, regardless of the Intermediaries' own bias.

If any of these conditions fails, the Sender may not be able to achieve her feasible first best, and may try to mitigate that inferior outcome by reverting to self-censoring or by restricting communication to a single Intermediary. 
Specifically, if the Intermediaries have similar preferences as to what the final Receiver should know, then there are "collusive" equilibria that tilt the composition of information in this direction (although competitive equilibria still exist). Secondly, if the communication capacities of the Intermediaries are asymmetric, then the superior communicator will be able to obtain an "eloquence rent" by tilting the message towards his priories. Finally, if the Sender is not benevolent, then she may find it optimal to prevent competition between Intermediaries. Inviting an Intermediary with similar preferences to an exclusive interview may help to tilt the message towards what the Sender likes.

The received literature has observed that the outcome in communication games depends on preference differences between the players (for example, Crawford and Sobel, 1982). The main contribution of this study is to point out that communication constraints are as important as preference misalignment in determining this outcome. The model below adopts a particularly mild form of preference misalignment - all agents want all other agents to learn what the state of nature is, although they have somewhat different priorities as to which issues are relatively more important. As a consequence of the communication constraints, an interesting interaction among agents emerges.

Perhaps the best example of the situation modeled in this paper is popularization of science. ${ }^{1}$ Firstly, the main concern of the scientists is to inform the public, rather than to manipulate them into an action that ignores their interest; this is also true for the science journalists. Secondly, the public is inattentive, which implies that nobody will ever be informed perfectly. ${ }^{2}$ More subtly, the public is heterogeneous in its inattention, namely, some segments of the public are more inattentive than others. ${ }^{3}$ Finally, research-active scientists are rarely directly involved in popularization of science. This forces them into a communication situation with limited control over the journalists that occupy the communication channel. ${ }^{4}$ In situations like this, experts

\footnotetext{
${ }^{1}$ The landmark Bodmer Report published in 1985 by the Royal Society is often credited as the first major investigation into how to improve public understanding of science.

${ }^{2}$ Bodmer Report (1985), page 21: "The whole process of reduction of the scientific information to a manageable form by the science journalist is almost bound to lead to some distortion(...)". Also, Nelkin (1995), pages 117-120.

${ }^{3}$ Bodmer Report (1985), page 14. More recently, there has been a lot of research on the related concept of information gap hypothesis in mass communication (e.g. Gaziano (2016)), which is defined as the difference in the rate of information acquisition by audiences with different socioeconomic status.

${ }^{4}$ Bodmer Report (1985), page 21: "If scientists are to communicate with the public through the media, they must, however, learn to accept the media's constraints and to convey information on the journalists' terms."
} 
may be strategic in their communication. ${ }^{5}$

Other examples could include an expert in international relations communicating the state of conflict in various regions and the journalists having different opinion in regard to which regions matter; central banks issuing statements about the economy, ${ }^{6}$ while economic pundits dissenting as to which sector of the economy or what time horizon matters more; a doctor thinking that the recovery rate and the side effects of a new pharmaceutical are equally important, but a journalist being interested only in side effects; a trade expert discussing the overall costs and benefits of future trade deals, while the journalists focusing only on specific opportunities. Communication frictions in these examples do not arise necessarily because agents are conflicted with each other - although that may be the case as well-but because the communication constraint forces trade-offs that do not exist otherwise.

The framework developed in this paper can be used to conduct the following kind of thought experiments. Suppose that a new communication technique - for instance, online media-becomes available and is embraced by an early adopter. This exogenous shock can be represented as an improvement of the communication capacity of one Intermediary, giving him a dominant position that tilts the content of communication to his advantage. The Sender might react by resorting to self-censorship, particularly if the preferences of the Sender and the dominant Intermediary are sufficiently misaligned. However, as soon as the other Intermediary catches up, the early adopter's advantage disappears and, in particular, the benevolent Sender can stop worrying about the influence of a single Intermediary.

The communication constraint is the main innovation of this paper and the key factor responsible for the results. Although this study uses a reducedform communication constraint, its deeper foundations are discussed in section 4. Sincerity and self-censorship are not the only policies that the Sender might potentially find useful, but it is also asserted that the richer space of policies does not cause any problems in the examples considered, and, therefore, the investigation presented in the first part is valid. ${ }^{7}$

The objective of the current paper is to study flexible communication, without committing to any specific communication protocol. Only the minimal assumption on communication is imposed, that the sender's ability to

\footnotetext{
${ }^{5}$ See Post (2016) who goes beyond the straightforward advice to withhold information that would be harmful to the public (Nelkin, 1995, pages 149-158).

${ }^{6}$ Blinder at al. (2008) survey both theoretical and empirical research on central bank communication.

${ }^{7}$ This analysis is based on communication constraints derived from entropic cost assumptions. Section 4 reports only the conclusions of this investigation but the details are available on request.
} 
reduce the receiver's uncertainty about the state of nature is limited. Within this constraint, the sender can shape her message, and the resulting outcome, anyway she wants. Such flexibility is a double-edged sword, as it also gives the Intermediary an equivalent power, as he may repackage the Sender's message in any way he wants.

Even with this flexibility, however, the Intermediary cannot reveal what he does not know. More generally, the measure of variance and co-variance between the issues in the Intermediary's communication is bounded below by the corresponding measure used by the Sender in her communication. In the world of full flexibility, it is only through this constraint that the Sender can influence the behavior of the Intermediary.

This paper connects to two strands of literature, the first of which is on games of communication. The soft communication (cheap talk) approach adopts the assumption of the seminal paper by Crawford and Sobel (1982) that facts may be costlessly misreported. The verifiable (hard talk) approach assumes that information cannot be lied about, although it can be withheld. ${ }^{8}$ This study is closer to the second approach by assuming that the Sender publicly commits to an editorial policy prior to the realization of the state of nature and prior to other decisions.

In a more applied context, communication models have been employed in studies of markets for news. In an essay reviewing the role of competition in those markets, Gentzkow and Shapiro (2008) caution against applying economic intuition from product markets to information markets. However, if there is a friction in processing information, then these differences are blurred. Various sources of information effectively become imperfect substitutes, leading to an analysis of competition similar in spirit to the one in product markets. The competition results of the current study echo Milgrom and Roberts (1986) and Mullainathan and Shleifer (2005) who show that if agents are exposed to information from sufficiently diverse sources then they can form accurate beliefs.

The second strand of the relevant literature is on rational inattention, which uses tools of information theory (Shannon, 1948) to study cognitive frictions in decision problems; see Sims (2003), (2006) and the macroeconomic literature that emerged since then. In recent years, a number of articles studying flexible information acquisition were published. ${ }^{9}$ In contrast to

\footnotetext{
${ }^{8}$ For example, Milgrom (1981), Milgrom and Roberts (1986), Admati and Pfleiderer (1986), Hörner and Skrzypacz (2016), Kamenica and Gentzkow (2011).

${ }^{9} \mathrm{~A}$ foundational paper by Matějka and McKay (2015) focuses on general properties of a decision problem when information can be acquired at an entropic cost; Matějka et al. (2017) apply similar tools to a dynamic decision problem. Yang (2015) has used this type of behavioral constraint in studying coordination and global games when information
} 
these models, the current paper studies flexible communication rather than flexible information acquisition - it is the sender, not the decision maker, who shapes what the decision maker learns. Agents are not viewed as boundedly rational; they are merely impatient in their role as readers of messages. ${ }^{10}$

\section{Model}

This section introduces a model of communication with simplified communication constraints. Some assumptions are questioned later in the paper - the communication constraint is investigated further in section 4, while other assumptions are discussed in the closing section 5 .

\section{Agents and states}

Let $X_{1}$ and $X_{2}$ be two independent standard normal random variables. In other words, the distribution of $X=\left(X_{1}, X_{2}\right)$ is $N(0, \boldsymbol{K})$, where $\boldsymbol{K}$ is an $2 \times 2$ identity co-variance matrix. The realization of $X$ is a state of nature, and each dimension of $X$ is called an issue.

There are three types of players: the Sender, the Intermediaries and the final Receivers. The Sender knows the realization of $X$. There are two types of final Receivers - more attentive and less attentive. Based on the messages that the final Receivers observe, they will form estimates of the true state $X$. Let the more attentive final Receiver's estimate be denoted $Y$, and let the less attentive final Receiver's estimate be denoted $Z$. Both $Y$ and $Z$ are 2-dimensional.

The top panel of Figure 1 shows this communication structure schematically. The key assumption and the source of all communication problems is that the Sender can communicate to the final Receivers only via the Intermediaries.

\section{Preferences}

The functional forms of preferences reflect two features. Firstly, all agents agree that more knowledge among all receivers is better than less, namely,

acquisition is costly but flexible. Denti (2017) investigates games in which players can learn not only about the exogenous state of nature but also about information of other players: sufficiently flexible learning, with entropic cost of information, is a key ingredient of his analysis.

${ }^{10}$ To my knowledge little has been published in economics on communication using information theory. An exception is a paper on organizational focus by Dessein at al. (2016), which does not, however, exploit any special features of flexible communication. 

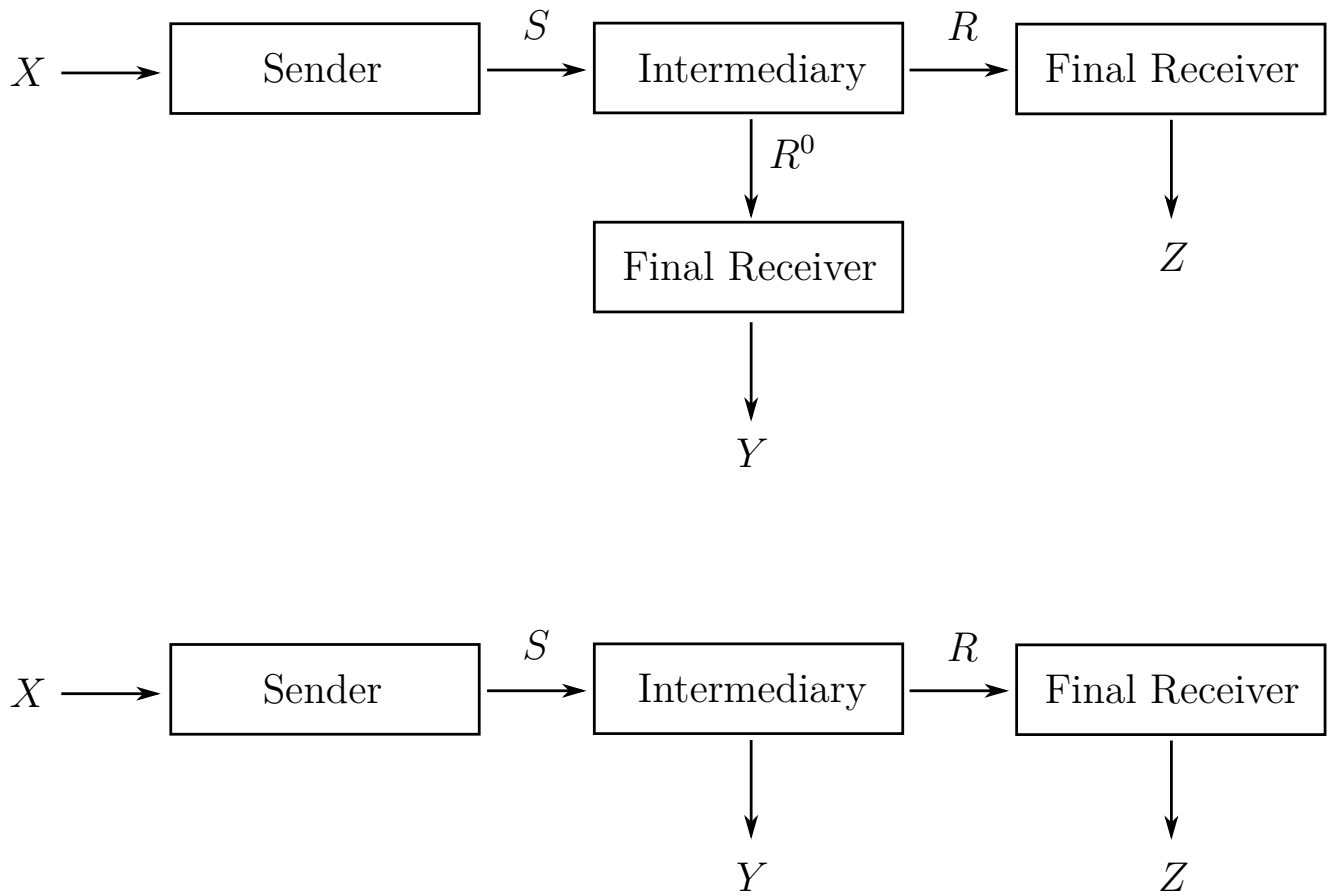

Figure 1: Communication structure

that the realizations of random variables $X_{k}-Y_{k}$ and $X_{k}-Z_{k}$ should be as close to zero as possible, and that nothing else matters. ${ }^{11}$ Secondly, agents may disagree as to which issue $k$ is relatively more important.

Formally, the first feature is captured by assuming that agents act to minimize the expected square difference between the state and its estimate, henceforth called a distortion. That is, if we denote the variance of the random variable $X_{k}-Y_{k}$ as $\Sigma_{k}$, then the distortion associated with the more attentive final Receiver's guess of issue $k$ is $\Sigma_{k}$. Likewise, if the variance of $X_{k}-Z_{k}$ is denoted $\Phi_{k}$, then the distortion associated with the less attentive final Receiver's guess of issue $k$ is $\Phi_{k}$. Define vectors $\Sigma=\left(\Sigma_{1}, \Sigma_{2}\right)$ and $\Phi=\left(\Phi_{1}, \Phi_{2}\right)$.

The second feature of preferences is captured by assuming that agents put different weights on different issues. Specifically, assume that the Sender's loss function is linear, $L_{S}(\Sigma)=\alpha \Sigma_{1}+\Sigma_{2}$, and that $\alpha>0$ is a weight that the Sender puts on issue 1 (slightly abusing notation, we will write this as the dot product, $\alpha \cdot \Sigma=\alpha \Sigma_{1}+\Sigma_{2}$.) The ultimate loss of the Sender is a

\footnotetext{
${ }^{11}$ In this sense, everyone is altruistic; experts and journalists are intrinsically motivated to reveal what they know. In particular, neither the Sender nor the Intermediaries have any pecuniary motives in attracting advertisers or audience, nor they want to induce a particular action, such as buying a product or voting for a candidate.
} 
combination of the losses from two types of the final Receivers,

$$
\lambda L_{S}(\Sigma)+(1-\lambda) L_{S}(\Phi)
$$

where $0 \leq \lambda \leq 1$ is a weight that the Sender puts on the more attentive final Receiver.

The Intermediary, in his role of a sender of information to the final Receivers, would also like the distortions to be as close to zero as possible. The particular form of his loss function is

$$
L_{I}(\Phi)=\beta \cdot \Phi
$$

where $\beta>0$ is the Intermediary's weight on issue 1 . Whenever there are two Intermediaries, the preference parameter of Intermediary $i$ will be indicated by a superscript, $\beta^{i}$.

Lastly, the final Receivers have their own weight $\gamma>0$ to measure which issue matters. Namely, their loss function is

$$
L_{R}(\Phi)=\gamma \cdot \Phi
$$

Although one can derive all the results for arbitrary preference parameters $\alpha, \beta^{i}$ and $\gamma$, it does make sense to assume some interpretable structure. I will say that the Sender is benevolent if her preferences are aligned with the preferences of the final Receiver, $\alpha=\gamma$. It is the Intermediaries who have different preferences than the final Receivers, $\beta^{i} \neq \alpha=\gamma$, in which case I will say that they are biased. Assuming without loss of generality that $\beta^{1}<\beta^{2}$, I will talk about two-sided bias if $\beta^{1}<\gamma<\beta^{2}$, and one-sided bias if either $\beta^{1} \leq \beta^{2}<\gamma$ or $\gamma<\beta^{1} \leq \beta^{2}$. I tend to think of the benevolent Sender and biased Intermediaries as the benchmark case, but other cases are interesting too. $^{12}$

\footnotetext{
${ }^{12}$ The expert and the public could differ in their opinion as to what matters, $\alpha \neq \gamma$. In one interpretation, the expert just wants to talk about her hobby, and thus is not benevolent; in another interpretation, a paternalistic Sender might have the best interests of the public in view, but the public might be thinking incorrectly that an issue is important when in fact it is not. Since the model is agnostic as to what issues the public ought to be interested in, this interpretation is problematic. Instead of appealing to such notion of paternalism, perhaps it would be better to frame this situation as an externality problem, whereby the public's knowledge about an issue has a positive effect on the public at large (see, for example, the discussion "Hard News vs. Soft News" in Gentzkow and Shapiro, 2008). The members of the public, acting independently, would under-demand information on this issue, but the expert could take this positive externality into account and counteract.
} 


\section{Communication friction}

The key premise of this study is that all players are inattentive as information receivers or they are ineloquent as senders. I focus on general statistical properties of the equilibrium behavior, therefore the details of communication protocols are not important. It is presumed that the set of those protocols is rich enough to generate the set of feasible distortions presented in the next few paragraphs. Any such feasible informational policy can be used by senders at no cost.

I assume specific functional forms for these constraints (section 4 shows that these forms can be derived from more fundamental plausible assumptions). Specifically, in all instances of communication, the communication constraint says that the products of distortions across issues cannot be lower than an exogenous capacity parameter. For example, $S \leq \Sigma_{1} \Sigma_{2}$ means that the receivers' distortion point $\left(\Sigma_{1}, \Sigma_{2}\right)$ cannot be too close to zero. Here, the exogenous $S \geq 0$ describes the level of communication friction, such as the attention of the receiver or the eloquence of the sender. The closer this number to zero, the better communication is possible. Moreover, there is a tradeoff between issues in communication, namely, once the boundary of feasible communication is achieved, one can make $\Sigma_{1}$ lower only by correspondingly increasing $\Sigma_{2}$. While the particular form of this constraint appears to be arbitrary, it seems to capture a fundamental communication friction. The set of points $\Sigma$ that satisfy this constraint with equality is called the sender's communication frontier. ${ }^{13}$

To be more specific, the top panel of Figure 1 shows all stages of communication and the corresponding capacity rates. The rate of communication between the Sender and the Intermediary is $S$, between the Intermediary and the more attentive final Receiver is $R^{0}$, and between the Intermediary and the less attentive final Receiver is $R$, where $R^{0}<R$. Thus, if the distortions profiles $\left(\Sigma_{1}, \Sigma_{2}\right)$ and $\left(\Phi_{1}, \Phi_{2}\right)$ are to be communicable between the Sender and the Intermediary, we must have $S \leq \Sigma_{1} \Sigma_{2}$ and $S \leq \Phi_{1} \Phi_{2}$. If $\left(\Sigma_{1}, \Sigma_{2}\right)$ is to be communicable from the Intermediary to the more attentive final Receiver, we must have $R^{0} \leq \Sigma_{1} \Sigma_{2}$, and, finally, if $\left(\Phi_{1}, \Phi_{2}\right)$ is to be communicable to the less attentive final Receiver, we must have $R \leq \Phi_{1} \Phi_{2}$.

This study focuses on the configuration $R^{0} \leq S<R$, which represents the case in which only the less attentive final Receiver is less attentive than

\footnotetext{
${ }^{13}$ The functional form is not very important. Any strictly convex frontier would lead to the same qualitative results, although the specific formulae derived below would be different. However, the multiplicative form of the communication frontier emerges directly from the entropic cost assumption discussed in section 4, so it has a reasonably strong justification.
} 
the Intermediary. The Intermediary faces a trivial decision problem when designing his communication to the more attentive Receiver - the optimal thing to do is to simply forward the messages from the Sender. However, the Intermediary faces a nontrivial compression problem when communicating to the less attentive final Receiver, as not enough bandwidth is available to repeat the messages from the Sender. ${ }^{14}$

In short, this simplifies the communication structure into the form depicted schematically in the bottom panel of Figure 1. There are two stages in which communication is designed. Stage one is communication from the Sender to the Intermediaries, where $\left(\Sigma_{1}, \Sigma_{2}\right)$ is determined, and stage two is communication from the Intermediary to the less attentive final Receiver, where $\left(\Phi_{1}, \Phi_{2}\right)$ is determined. The relevant constraint in stage one and two, respectively, are

$$
\begin{aligned}
& S \leq \Sigma_{1} \Sigma_{2} \\
& R \leq \Phi_{1} \Phi_{2}
\end{aligned}
$$

There is also a second set of communication constraints, but they are rather uncontroversial. Point $\left(\Sigma_{1}, \Sigma_{2}\right)$ must obey

$$
0 \leq \Sigma_{k} \leq 1
$$

for $k=1,2$. It says that, no matter what communication policy is selected, the distortion cannot be made greater than what it already is without any communication (the maximal distortion is equal to the variance of $X_{k}$, which is one.) Finally, point $\left(\Phi_{1}, \Phi_{2}\right)$ must obey

$$
\begin{aligned}
& \Phi_{k} \leq 1 \\
& \Sigma_{k} \leq \Phi_{k}
\end{aligned}
$$

for $k=1,2$. This says that, in addition to not increasing the distortion beyond what it is without communication, the Intermediary cannot communicate more than what he knows. Namely, the distortion that he induces, $\Phi_{k}$, cannot be lower than $\Sigma_{k}$.

Constraint (8) will be referred to as the constraint from below. This is to contrast it with constraints like (7) and (6) which will be called constrains from above. Throughout this paper, I am not going to be focused on constraints from above. They must hold, of course, but their presence does not add much to the core arguments. In most intuitive explanations I will simply

\footnotetext{
${ }^{14}$ This is the reason why the loss function of the Intermediary in equation (2) is defined only on the distortion on the less attentive final Receiver. In principle, it could have the form $\lambda L_{I}(\Sigma)+(1-\lambda) L_{I}(\Phi)$, like the loss function of the Sender, but since the first term is independent of the Intermediary's action, there is no loss from ignoring it.
} 
assume that the parameters are such that they are not binding. For example, it would be enough to assume that $S$ and $R$ are sufficiently close to zero. Constraints from below (8), on the other hand, will play a critical role.

If there are two Intermediaries present, I assume that both of them are equally capable of listening to the Sender at the common capacity $S$. All variables that pertain to Intermediary $i$ will have a superscript $i$, such as endogenous variables $\Phi^{i}$, or exogenous $\beta^{i}$ and $R^{i}$, where the latter is the communication rate between the Intermediary $i$ and the less attentive final Receiver.

\section{Extensive form game and the solution concept}

A simple, complete information, dynamic game in informational policies is considered. In stage one, the Sender decides which of the two Intermediaries to inform, or both. She then chooses her informational policy $\Sigma$, common for the selected Intermediaries. Her objective function is (1) and constraints are (4) and (6). In the second stage, the selected Intermediaries learn $\Sigma$ and simultaneously choose their respective informational policies $\Phi$. The objective function of each is (2) and constraints are (5), (7) and (8). If only one monopolistic Intermediary is selected by the Sender, then the game ends, and, in particular, the final Receiver remains a passive information consumer. If two Intermediaries are selected, then there is a third stage in which the final Receiver is an active decision maker. She selects an Intermediary based on her objective function (3). Assume a tie-breaking rule operating when the final Receiver is indifferent between the Intermediaries: she selects the one with the better capacity $R^{i}$, and if these are the same, then she selects one randomly. When all informational policies are in place, communication plays out automatically according to these policies, and payoffs are realized.

The solution concept is subgame perfect Nash equilibrium.

\section{Analysis}

Before turning to the analysis of the game between independent players, this section starts by considering a hypothetical situation in which the Sender has full control over the policy of a single Intermediary. I will refer to this as the dictator problem. This scenario is not the main case of interest, but it is presented here as one benchmark. 


\subsection{Dictator}

The dictator's problem is to select $\Sigma$ and $\Phi$ to maximize (1), subject to the battery of constraints (4), (6), (5), (7) and (8).

The dictatorial distortions can be calculated via the standard KuhnTucker method. If one assumes that $R \leq \alpha \leq 1 / R$, then the constraints from above are not binding and the solution is $\Sigma^{*}=(\sqrt{S / \alpha}, \sqrt{S \alpha})$ and $\Phi^{*}=(\sqrt{R / \alpha}, \sqrt{R \alpha})$.

The left panel of Figure 2 illustrates the optimal choice of such a dictator. The two axes measure distortions of issue one and two, respectively, so point $(0,0)$ represents perfect communication, while $(1,1)$ represents no gains from communication whatsoever. Curves $S$ and $R$ represent communication frontiers (4) and (5), respectively; distortion points are feasible if and only if they lie on or above the respective curve. The tangent lines of slope $\alpha$ are indifference curves of the dictatorial Sender. More generally, the dashed ray of slope $\alpha$ indicates the expansion path, namely the locus of optimal points for various capacity levels, such as $S$ or $R$. Points $\Sigma^{*}$ and $\Phi^{*}$, which are the solution to the dictator problem, are indicated too. ${ }^{15}$

\subsection{One Intermediary}

Now, I turn to the case of Intermediaries as independent players. In this subsection, I consider a subgame in which the Sender selects a single Intermediary characterized by his eloquence $R$ and preference parameter $\beta$. Without loss of generality, let $\alpha>\beta$, so that the Intermediary cares about issue one relatively less than the Sender.

The right panel of Figure 2 illustrates the problem of the Sender facing an independent Intermediary. As before, the Sender's communication frontier is shown as curve $S$, while the one for the Intermediary is shown as $R$. The dashed rays with slopes $\alpha$ and $\beta$ are expansion paths for preferences $\alpha$ and $\beta$, respectively. The Figure and the calculations below are based on parameters $\alpha=1, \beta=0.4, S=0.04, R=0.2$ and $\lambda=0.5$. For this configuration of parameters, the dictatorial Sender would select point $\Sigma^{s}=(0.2,0.2)$ in stage one and point $\mathrm{M} \approx(0.45,0.45)$ in stage two.

\footnotetext{
${ }^{15}$ The Sender may have different preference weights across stages, say, $\alpha^{1}$ in stage one and $\alpha^{2}$ in stage two. If the Sender cared about issue two in stage two more than in stage one, $\alpha^{2}<\alpha^{1}$, then the solution in stage two, that is, point $\Phi^{*}$, would move along the communication frontier (5) to the right, towards point C. In the extreme case, when $\alpha^{2}$ becomes lower than $\alpha^{1} S / R$, the preferred choice in stage two would "like" to move to the right of point $\mathrm{C}$, but this would be impossible, as one of the constrains from below (8) would become binding.
} 

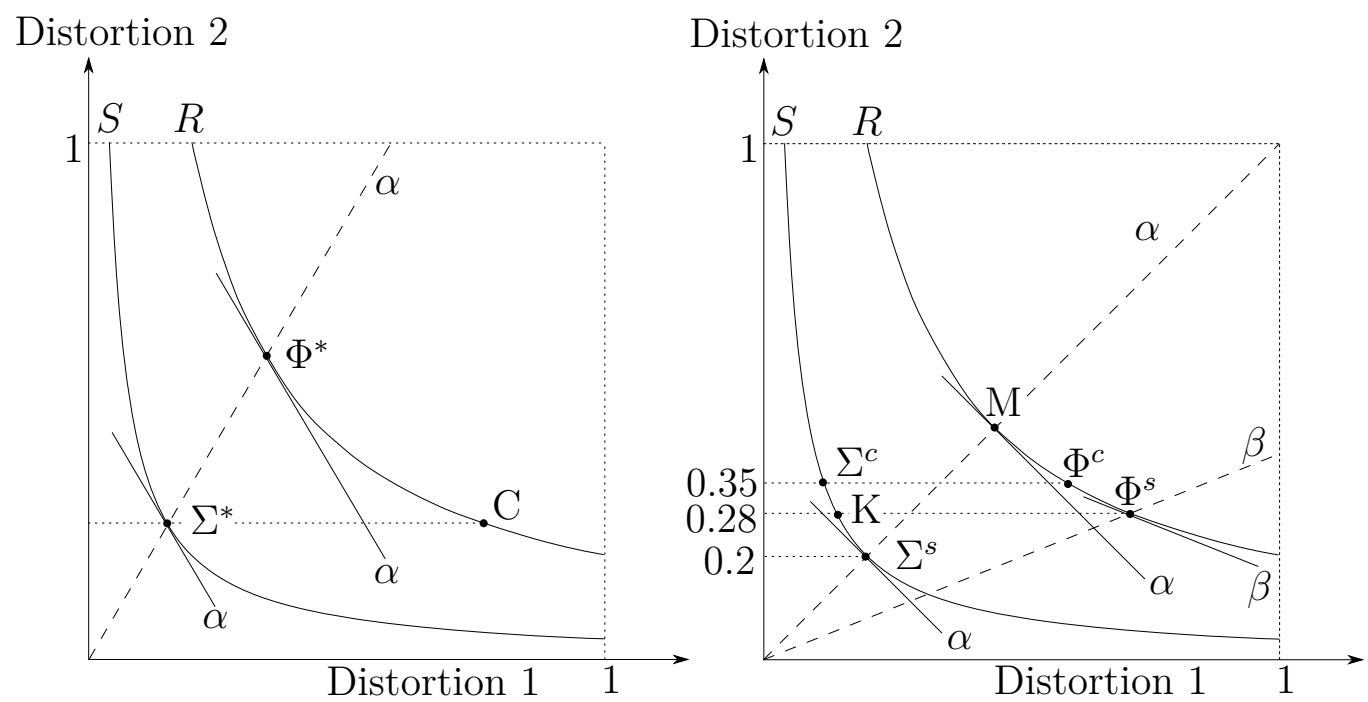

Figure 2: Left: dictatorial policy. Right: one independent Intermediary.

The Sender facing an independent Intermediary can mimic the dictatorial Sender by selecting the same $\Sigma^{s}$ in stage one. However, the independent Intermediary would not select $\mathrm{M}$ as a best response. He has both an opportunity and incentives to select something else. Since $\beta<\alpha$, the Intermediary wants to focus more on issue two, relative to the Sender. Consequently, the best response of the Intermediary to $\Sigma^{s}$ is $\Phi^{s}=(0.71,0.28)$ which lies on the $\beta$ expansion path. Obviously, from the Sender's point of view, this outcome is worse than the dictatorial one, and the greater the difference between $\beta$ and $\alpha$, the greater this loss is.

The Sender may select a different distortion point than $\Sigma^{s}$. Suppose that she trades-off distortion in issue one for distortion in issue two, along her stage-one communication frontier $S$. Graphically, she moves the stage-one distortion point from $\Sigma^{s}$ towards point $K$. If that deviation is small, the Intermediary can still select the same point as before, $\Phi^{s}$. The resulting overall outcome is clearly worse for the Sender than the sincere policy $\Sigma^{s}$. However, if the Sender moves past point $\mathrm{K}$, the Intermediary runs into a constraint from below, (8), so he is no longer able to select point $\Phi^{s}$. He is forced to increase the stage-two distortion in issue two, reducing distortion in issue one, which is unambiguously beneficial for the Sender. To see whether this departure from $\Sigma^{s}$ is beneficial overall, one must measure a better outcome in stage-two against a worse outcome in stage-one.

Moving on to formal results, the equilibrium strategies of the Intermediary and the Sender are presented in Lemma 1 and Proposition 1, respectively. Let $B(R, \beta)$ denote the best distortion point of an Intermediary with $(R, \beta)$, 
in response to the Sender's policy $\Sigma$. Namely,

$$
B(R, \beta)=\arg \min _{\Phi: \Sigma \leq \Phi \leq 1, R \leq \Phi_{1} \Phi_{2}} \beta \cdot \Phi
$$

Taking advantage of the functional forms of the loss function and the feasible set, we can calculate this best response explicitly. To avoid complications associated with constraints from above, assume that $R \leq \beta \leq 1 / R$ and $S \leq \alpha \leq 1 / S$.

Lemma 1. Suppose the Sender announces policy $\Sigma$. If $R \leq \Sigma_{1} \Sigma_{2}$ then the best response of the monopolistic Intermediary is $B(R, \beta)=\Sigma$. Otherwise, if $\Sigma_{1} \Sigma_{2}<R$, it is

$$
B(R, \beta)= \begin{cases}(\sqrt{R / \beta}, \sqrt{\beta R}) & \text { if } \Sigma_{1} \leq \sqrt{R / \beta} \text { and } \Sigma_{2} \leq \sqrt{R \beta} \\ \left(R / \Sigma_{2}, \Sigma_{2}\right) & \text { if } \Sigma_{2}>\sqrt{R \beta} \\ \left(\Sigma_{1}, R / \Sigma_{1}\right) & \text { if } \Sigma_{1}>\sqrt{R / \beta}\end{cases}
$$

Appendix A contains all proofs.

The first line of (9) shows what happens when constraint (8) is not binding, and therefore the Intermediary's best response is on his unconstrained expansion path; the second and third lines show the case when constraint (8) is binding and the Intermediary is forced to select a different distortion point than on his unconstrained expansion path.

Consider now the behavior of the Sender. Her dilemma is whether to engage in trying to affect the Intermediary's behavior via constraint (8). Define two specific policies. The policy $\Sigma^{s}=(\sqrt{S / \alpha}, \sqrt{S \alpha})$, will be called sincere because it is optimal for the dictator in stage one, as if the Intermediary's plans what to do in stage two did not matter. The policy $\Sigma^{c}=(S / \sqrt{T \alpha}, \sqrt{T \alpha})$, where $T=\lambda S+(1-\lambda) R$, is called self-censoring because the sender limits his communication of one issue in stage one in order to prevent the intermediary from focusing on this issue in stage two.

Proposition 1. The Sender's optimal policy satisfies her communication constraint with equality $\Sigma_{1} \Sigma_{2}=S$. Furthermore, it depends on the measure of preference similarity, $\beta / \alpha<1$, relative to parameters $S, R$ and $\lambda$ :

1. If preferences are close to each other, $T / R \leq \beta / \alpha \leq 1$, then the optimal policy is sincere, $\Sigma^{s}$.

2. If preferences are more distant, $S / R<\beta / \alpha<T / R$, then both $\Sigma^{c}$ and $\Sigma^{s}$ are local optima. The overall optimal policy is the sincere policy $\Sigma^{s}$ if

$$
\frac{1}{\sqrt{\beta / \alpha}}+\sqrt{\beta / \alpha} \leq 2 \frac{\sqrt{T}-\lambda \sqrt{S}}{(1-\lambda) \sqrt{R}}
$$


which holds for $\beta / \alpha$ high enough. Otherwise, it is self-censorship $\Sigma^{c}$.

3. If preferences are even more distant, $\beta / \alpha \leq S / R$, then the optimal policy is self-censorship, $\Sigma^{c}$.

The right panel of Figure 2 depicts both candidate solutions for Sender, $\Sigma^{s}=(0.2,0.2)$ and $\Sigma^{c}=(0.12,0.35)$. The best response to $\Sigma^{s}$ is the already mentioned $\Phi^{s}=(0.71,0.28)$, by the first line of $(9)$. The best response to $\Sigma^{c}$ is $\Phi^{c}=(0.58,0.35)$, by the second line of $(9)$. As it happens, inequality (10) does not hold, implying that the outcome of self-censoring $\left(\Sigma^{c}, \Phi^{c}\right)$ is indeed better for the Sender than the outcome of the sincere policy $\left(\Sigma^{s}, \Phi^{s}\right)$.

There are a few insights from this analysis. Firstly, the flexibility assumption is a double-edged sword for the Sender. Even if it allows her to manipulate her communication, it also allows the Intermediary to completely "undo" this manipulation, as long as the constraint (8) is not binding. Secondly, this constraint, if binding, is the only channel through which the Sender can successfully affect the behavior of the Intermediary. The Sender uses self-censorship here not because she wants to hide information harmful to her, but rather to prevent the Intermediary from over-communicating issues she thinks are not as important as some other issues. The choice between sincere and self-censoring policy has also distributional consequences for the receivers. Sincere policy targets more attentive audience. Self-censorship is more "populist" in the sense of focusing on the less attentive audience at the expense of providing inferior information to the more attentive audience.

\subsection{Two Intermediaries}

This subsection considers a subgame in which the Sender selected both Intermediaries to communicate to, where Intermediary $i=1,2$ is characterized by $\left(R^{i}, \beta^{i}\right)$. This configuration triggers a nontrivial stage game between the Intermediaries who now compete for the attention of the less attentive final Receiver. Without loss of generality, assume that Intermediary 1 is a better communicator, $S<R^{1} \leq R^{2}$, and that he cares about issue 2 more, so that his indifference lines are flatter, $\beta^{1} \leq \beta^{2}$.

Both panels of Figure 3 illustrate this configuration of parameters. As before, the axes measure distortions. The curves denoted $R^{1}$ and $R^{2}$ indicate communication frontiers of Intermediary 1 and 2, respectively (the Sender's communication frontier is not depicted). The dashed rays, such as $\beta^{1}, \beta^{2}$ and $\gamma$ are expansion paths for agents with these preference parameters, respectively. In other words, these are locations of optimal distortion points for these preference parameters, as communication capacities vary and there 

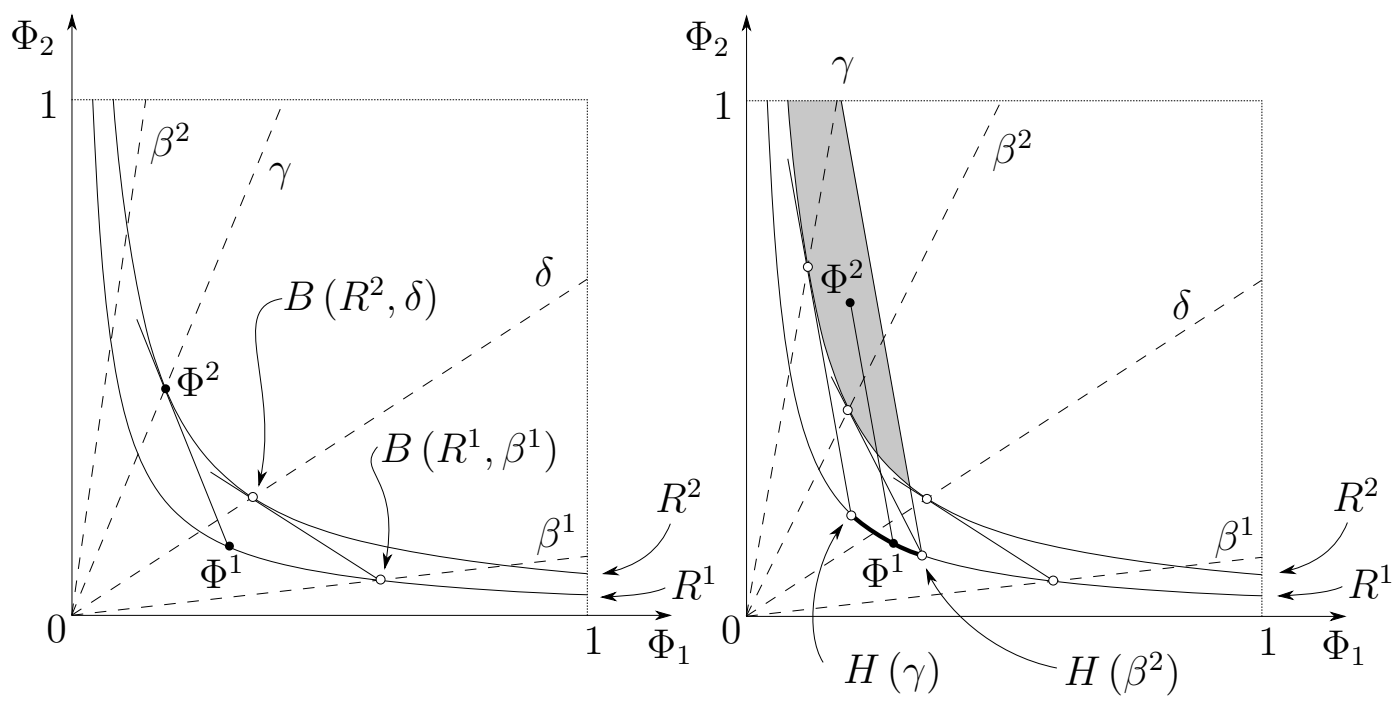

Figure 3: Case $\delta<\gamma$. Left: two-sided bias, $\beta^{1}<\gamma \leq \beta^{2}$. Right: one-sided bias, $\beta^{1}<\beta^{2}<\gamma$. Set $\Delta$ is shaded.

are no other constraints. The panel on the left depicts the two-sided bias case, $\beta^{1}<\gamma<\beta^{2}$, and the one on the right shows the one-sided bias case, $\beta^{1}<\beta^{2}<\gamma$.

Both panels of Figure 3 show the case in which the more capable communicator, Intermediary 1 , is sufficiently biased. To understand what this means, define a threshold $\delta$ to be a positive scalar that satisfies

$$
\delta \cdot B\left(R^{2}, \delta\right)=\delta \cdot B\left(R^{1}, \beta^{1}\right)
$$

The left panel of Figure 3 shows that parameter $\delta$ can be imagined as a preference parameter of a hypothetical agent who is indifferent between distortion point $B\left(R^{2}, \delta\right)$ (the best available to him at $\left.R^{2}\right)$ and $B\left(R^{1}, \beta^{1}\right)$ (the one that is most preferred by Intermediary 1$)$. Notice that $\delta$ depends only on exogenous parameters $\left(R^{1}, \beta^{1}\right)$ and $R^{2}$ (specifically, $\beta^{1} \leq \delta$ ), but it is independent of $\gamma$ and $\beta^{2}$.

The first result considers the case that is complementary to the one depicted in Figure 3. That is, Intermediary 1 is not sufficiently biased, in the sense that $\gamma$ is closer to $\beta^{1}$ than threshold $\delta$, or simply $\gamma \leq \delta$.

Proposition 2. Let $\gamma \leq \delta$. If Intermediary 1 chooses $B\left(R^{1}, \beta^{1}\right)$, then this distortion point is selected by the less attentive final Receiver regardless of what Intermediary 2 chooses. Thus, $B\left(R^{1}, \beta^{1}\right)$ is a strictly dominant strategy for Intermediary 1. 
The rest of the analysis considers the more interesting case of $\delta<\gamma$, when Intermediary 1 is sufficiently biased relatively to $\gamma$.

Let $G(\gamma, D)$ be a feasible distortion point that is best for Intermediary 1, like $B\left(R^{1}, \beta^{1}\right)$, but with an extra constraint, which says that a hypothetical player with a preference parameter $\gamma$ prefers this point to a given reference distortion point $D$. Formally,

$$
\begin{gathered}
G(\gamma, D)=\arg \min _{\Phi: \Sigma \leq \Phi \leq 1, R^{1} \leq \Phi_{1} \Phi_{2}} \beta^{1} \cdot \Phi \\
\text { s.t. } \gamma \cdot \Phi \leq \gamma \cdot D
\end{gathered}
$$

Moreover, let $H(\gamma)$ be this distortion when arbitrary $D$ is replaced by the best outcome available to agent $\gamma$ at communication capacity $R^{2}$, namely, $H(\gamma)=G\left(\gamma, B\left(R^{2}, \gamma\right)\right)$.

Armed with these definitions, I can now present the set of equilibria and offer some graphical explanation. There are two propositions, the first one considers the two-sided bias, $\beta^{1}<\gamma \leq \beta^{2}$, while the next one deals with the last possible configuration of parameters, the one-sided bias, $\beta^{1} \leq \beta^{2}<\gamma$.

Proposition 3. Let $\delta<\gamma$. In the case of two-sided bias $\left(\beta^{1}<\delta<\gamma \leq \beta^{2}\right)$, the pair of distortions $\Phi^{2}=B\left(R^{2}, \gamma\right)$ and $\Phi^{1}=H(\gamma)$ forms a unique stagetwo equilibrium.

The left panel of Figure 3 illustrates the equilibrium in the two-sided bias case. Intermediary 1 selects point $\Phi^{1}$ and Intermediary 2 selects point $\Phi^{2}$. The less attentive final Receiver is indifferent between these two points, because $\gamma \cdot \Phi^{1}=\gamma \cdot \Phi^{2}$, and selects $\Phi^{1}$ by the tie-breaking rule. Point $\Phi^{1}$ is a best response to $\Phi^{2}$ by construction of $H(\gamma)$. In particular, selecting another feasible point to the right of the line going through $\Phi^{1}$ and $\Phi^{2}$ causes the final Receiver to switch to $\Phi^{2}$, which makes Intermediary 1 worse off; moving to the left of this line keeps the final Receiver but makes the final outcome only worse. Secondly, Intermediary 2 offers a distortion point that is best for the final Receiver given his capacity, and even then the final Receiver does not select it. Thus, Intermediary 2 obviously cannot deviate from $\Phi^{2}$ profitably.

If bias is one-sided then there are multiple equilibria. To characterize them all, define a set of distortions $\Delta$, for $\beta_{2}<\gamma$, as

$$
\Delta=\left\{\Phi \in \mathbb{R}_{+}^{2}: \begin{array}{c}
\Sigma \leq \Phi \leq 1, R^{2} \leq \Phi_{1} \Phi_{2} \\
\gamma \cdot \Phi \leq \gamma \cdot H\left(\beta^{2}\right)
\end{array}\right\}
$$

These are all distortion points feasible at rate $R^{2}$, with an additional constraint, whereby the less attentive final Receiver is to obtain a better distortion point than $H\left(\beta^{2}\right)$ (recall that point $H\left(\beta^{2}\right)$ is the best distortion profile 
that Intermediary 1 can get, assuming that Intermediary 2 selects his feasible first best).

The significance of set $\Delta$ lies in the fact that any point in this set can be used to play the role of point $B\left(R^{2}, \gamma\right)$ in Proposition 3. Formally,

Proposition 4. Let $\delta<\gamma$. In the case of one-sided bias $\left(\beta^{1} \leq \beta^{2}<\gamma\right)$, for any $\Phi^{2} \in \Delta$, there is a stage-two equilibrium in which Intermediary 2 selects $\Phi^{2}$ and Intermediary 1 selects $\Phi^{1}=G\left(\gamma, \Phi^{2}\right)$. Moreover,

1. Suppose $\delta<\beta^{2}$. Then there is no stage-two equilibrium in which Intermediary 2 selects $\Phi^{2} \notin \Delta$.

2. Alternatively, suppose $\beta_{2} \leq \delta$. Then in any stage-two equilibrium in which Intermediary 2 selects $\Phi^{2} \notin \Delta$, intermediary 1 chooses point $G\left(\gamma, \Phi^{2}\right)=B\left(R^{1}, \beta^{1}\right)$.

The right panel of Figure 3 shows one possible equilibrium in the one-sided bias case. Set $\Delta$ is depicted as the shaded area. Pick any point $\Phi^{2}$ in this set and derive $\Phi^{1}=G\left(\gamma, \Phi^{2}\right)$ from it. The final Receiver is indifferent between $\Phi^{1}$ and $\Phi^{2}$, because $\gamma \cdot \Phi^{1}=\gamma \cdot \Phi^{2}$, and selects $\Phi^{1}$ by the tie-breaking rule. Intermediary 1 does not have any incentives to deviate from $\Phi^{1}$ for the same reason as in two-sided bias case. Intermediary 2 can also deviate: moving to the right of the line going through $\Phi^{1}$ and $\Phi^{2}$ does not have any effect as the final Receiver keeps selecting $\Phi^{1}$; moving to the left causes the final Receiver to switch to this new point, which makes Intermediary 2 worse off. This construction works for any $\Phi^{2}$ in set $\Delta$. If $\Phi^{2}$ is outside of $\Delta$, then Intermediary 2 can profitably deviate to his feasible first best.

This analysis implies that, in the one-sided bias case, any distortion point on the communication frontier of Intermediary 1 between points $H(\gamma)$ and $H\left(\beta^{2}\right)$ can be supported as an equilibrium outcome, and only those points. This set of all possible equilibrium outcomes is depicted in the right panel of Figure 3 as a bold section of $R^{1}$ communication frontier, between distortion points $H(\gamma)$ and $H\left(\beta^{2}\right)$.

Finally, let us record the following by-product of this analysis.

Corollary 1. If $R^{1}<R^{2}$, then in any equilibrium the final Receiver selects the more eloquent Intermediary 1.

\section{Comparative statics in the stage-two game}

The results contained in Propositions 2, 3 and 4 depend on exogenous parameters $R^{1}, R^{2}, \beta^{1}, \beta^{2}$ and $\gamma$. 


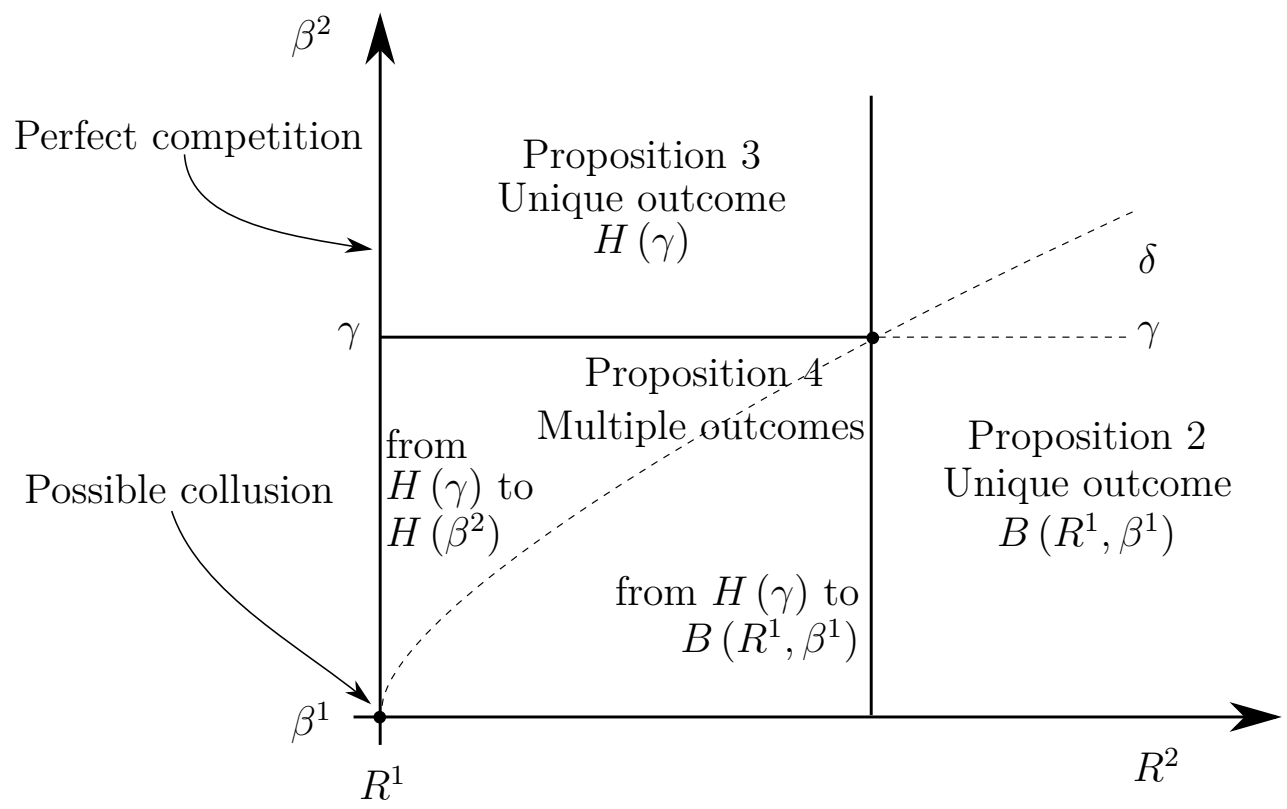

Figure 4: Comparative statics with two Intermediaries

Figure 4 shows the map of these results as a function of Intermediary 2's parameters $R^{2}$ and $\beta^{2}$ measured on the x-axis and y-axis, respectively. Intermediary 1's parameters, $R^{1}$ and $\beta^{1}$, are a fixed reference, represented by a point in the bottom-left corner of Figure 4. Parameter $\gamma$, also fixed, is indicated on the y-axis somewhere above $\beta^{1}$.

Threshold $\delta$ is a function of $R^{2}$ only, and it is represented by an increasing dashed curve starting at $\left(R^{1}, \beta^{1}\right)$. When $R^{2}$ is large enough, then $\delta$ becomes greater than a given $\gamma$, providing conditions for the dominant strategy equilibrium in Proposition 2. Otherwise, for lower $R^{2}$, the equilibria are described by Propositions 3 and 4 . Specifically, if $\beta^{2}$ is larger than $\gamma$, as in the top-left region, Proposition 3 describes the unique equilibrium. If $\beta^{2}$ is lower, the result is provided by Proposition 4, and the relevant region in Figure 4 is bottom left.

First, consider a Sender facing a communication problem with two features: the Intermediaries are equally capable in communication, $R^{1}=R^{2}$, and their heterogeneity of preferences is two-sided, $\beta^{1} \leq \gamma \leq \beta^{2}$. This case occurs in the top-left part of Figure 4, where it is described as perfect competition. The result is particularly striking in this case: the equilibrium outcome is unique and the best for the final Receiver, given the common communication constraint $R^{1}=R^{2}$.

In the logic somewhat similar to Bertrand price competition, Intermediaries engage in a "quality of information" competition. Instead of setting 
prices, they set their communication policies that determine a pair of distortion points from which the final Receiver chooses. They try to tilt the communication policy towards the issues they prefer, but in order to convince the final Receiver to select their distortion point, the policy has to be slightly better for the final Receiver than the one of their competitor. They continue approaching the best mix for the final Receiver until, inadvertently, they end up providing it. This stage-two equilibrium is completely unaffected by the magnitude of Intermediaries' biases, as long as their bias is two-sided.

Perfect competition may fail to materialize for two reasons. Firstly, Intermediary 2 may be a less capable communicator, giving some competitive advantage to Intermediary 1. Secondly, the preferences of the Intermediaries may be aligned, making their bias one-sided, and thus giving both Intermediaries a form of collusive power.

Let us consider the first of these two possibilities. A less eloquent Intermediary 2 is represented in Figure 4 as a move to the east from the region of perfect competition. The unique equilibrium outcome, $H(\gamma)$, diverges from the final Receiver's first best, $B\left(R^{1}, \gamma\right)$, and progressively shifts towards the Intermediary 1's first best $B\left(R^{1}, \beta^{1}\right)$. That point is achieved once $R^{2}$ becomes so great that it pushes $\delta$ above $\gamma$, and Intermediary 2 becomes irrelevant. Further increases of $R^{2}$ have no effect. ${ }^{16}$

The other way to depart from perfect competition is to make the preferences of the Intermediaries more aligned. Making preference parameter of Intermediary 2 closer to the one of Intermediary 1 , and keeping $R^{1}=R^{2}$, is represented in Figure 4 as a move south from the region of perfect competition, towards bottom-left corner, denoted as possible collusion. When $\beta^{2}$ becomes lower than $\gamma$, then more collusive equilibria emerge, alongside the competitive equilibrium. All distortion points on the $R^{1}$ communication frontier between the competitive one, $H(\gamma)$, and the most collusive one, $H\left(\beta^{2}\right)$, are equilibrium outcomes (see the right panel of Figure 3 ). At the end of this process, as $\beta^{2}$ converges to $\beta^{1}$, the Intermediaries become completely homogeneous in their capacity and preferences. The set of equilibria keeps increasing, until the best outcome for Intermediary 1 (and 2) becomes an equilibrium too. Perhaps it is not surprising that two Intermediaries whose interests are perfectly aligned achieve their best monopolistic outcome, $B\left(R^{1}, \beta^{1}\right)$. What is more surprising is that competitive outcomes remain equilibria.

\footnotetext{
${ }^{16}$ Continuing the analogy to the Bertrand price competition, Intermediaries with asymmetric communication capacities are similar to Bertrand sellers having asymmetric per unit costs. The higher the cost of one of the sellers, the closer the profit of the other seller is to the monopoly profit.
} 


\section{Sender's informational policy with two Intermediaries}

As far as the problem of the Sender in stage one is concerned, she can again engage in self-censorship.

In order to have a clear-cut discussion of self-censorship with two Intermediaries, let us focus on the case of a unique equilibrium, that is, on the case of two-sided bias. Let the stage-two equilibrium be $\left(\Phi^{1}, \Phi^{2}\right)$. Observe that point $\Phi^{1}$ lies to the south-east of point $\Phi^{2}$, that is, Intermediary 2 provides a lower distortion in the first issue, $\Phi_{1}^{1}>\Phi_{1}^{2}$, and greater distortion in the second issue, $\Phi_{2}^{1}<\Phi_{2}^{2}$. Suppose that, initially, constraints from below are not binding for any of the Intermediaries, like in Figure 3. Namely, both inequalities hold:

$$
\begin{aligned}
& \Sigma<\Phi^{1} \\
& \Sigma<\Phi^{2}
\end{aligned}
$$

Since there are two constraints now, the Sender can attempt to affect the final outcome in two ways. A direct way is by making constraint (12) binding. Self-censoring of issue 2 pushes the equilibrium point $\Phi^{1}$ to the north (and also west) along the $R^{1}$ communication frontier, via constraint $\Sigma_{2} \leq \Phi_{2}^{1}$. This is very similar to the case of a single Intermediary and Proposition 1.

An indirect way is by making constraint (13) binding. Specifically, the Sender may self-censor issue 1 through constraint $\Sigma_{1} \leq \Phi_{1}^{2}$, in order to push point $\Phi^{2}$ to the south (and also east) along the $R^{2}$ communication frontier. Of course, this distortion point is not itself consumed by the final Receiver, by Corollary 1, but the game between the Intermediaries in stage two is affected in such a way that Intermediary 1 moves his choice $\Phi^{1}$ along the $R^{1}$ communication frontier to the south-east too.

In the one-sided bias case, the stage-two equilibrium is not unique. However, the entire set of equilibrium outcomes can be controlled by the Sender's choice of $\Sigma$ in a similar way as in the two-sided bias case. ${ }^{17}$

\section{Foundation of the communication constraint}

The reduced-form communication constraints (4)-(8) play a critical role in the analysis. It is important to understand whether they can be backed up by some more fundamental arguments. The specific questions are "what exactly

\footnotetext{
${ }^{17}$ The equilibrium distortion points lie on the $R^{1}$ communication frontier between points $H(\gamma)$ and $H\left(\beta^{2}\right)$. The direct self-censoring of issue 2 moves the $H\left(\beta^{2}\right)$ end of this spectrum along the $R^{1}$ communication frontier to the north-west, while the indirect selfcensoring of issue 1 moves the $H(\gamma)$ end of this spectrum to the south-east along the $R^{1}$ communication frontier.
} 
is assumed in order to generate functional forms (4)-(8)?", and, more specifically, "is there any loss of generality in assuming (4)-(8)?" All assertions of this section have formal counterparts which are available upon request.

As soon as one assumes that communication is constrained, one has to propose how the senders compress their messages. One option is to use optimal compression techniques. Information theory, started by the seminal work of Shannon (1948), has established that an object called mutual information plays a fundamental role in measuring what can be communicated via a constrained communication channel. Roughly speaking, mutual information between $X$ and $Z$ measures the minimal length of the message so that action described by random variable $Z$ can be taken at the receiving end of communication in response to the realization of the source $X$. An elementary communication constraint states that mutual information is bounded by some exogenous rate of communication. ${ }^{18}$

Under the assumptions of this paper (Gaussian random variable $X$ with zero co-variance, and quadratic distortions), the first result is this: if the Sender uses Gaussian random variable $Y$ with zero co-variance, then it is optimal for the Intermediary to use Gaussian $Z$ and, moreover, constraints (4)(8) emerge exactly from the constraints that mutual information is bounded. This answers the first question posed above.

To shed light on the second question, one may investigate if the assumption made in the previous paragraph - the co-variance term in random variable $Y$ being zero - can be relaxed. That is, assume that the Sender, apart from having two degrees of freedom in selecting variances $\left(\Sigma_{1}, \Sigma_{2}\right)$, can also select the associated co-varinace term.

It turns out that if the loss function is linear, as in (1), then the predictions of the reduced-form communication model are correct. Specifically, the possibly non-zero co-variance term will be zero as the result of the optimal choice of the Sender, and the only possibly optimal policies of the Sender are indeed either sincere or self-censoring. However, different policies than these two may be optimal if the loss function (1) is not linear. For instance, if this loss function exhibits extreme complementarities across dimensions, it is possible to provide examples in which selecting a positive co-variance term generates a lower loss than either sincere or the best self-censoring policy.

\footnotetext{
${ }^{18}$ When communication involves an intermediary, like in the bottom panel of Figure 1, communication constraints are described by Yamamoto (1981), or by Theorem 20.4 in El Gamal and Kim (2011).
} 


\section{Conclusions and final remarks}

The main objective of this study is to investigate the implications of a plausible communication constraint, which reflects the fact that the senders are not eloquent enough and the receivers are not patient enough for communication to be completely frictionless. The preference differences across agents are minimal - everyone wants everyone to know more, although they have different priorities as to what aspect of reality should be communicated first. The analysis allows any type of hard communication policy, provided that it is flexible. The approach is indirect - I am not modeling the details of the communication protocol (like, for example, Alaoui and Germano, 2014), but I rather focus on general statistical properties of the equilibrium behavior, such as which facets of reality are communicated and what correlation profile between these separate issues is.

Flexibility is both a blessing and a curse for the Sender. She may shape her information policy to try to manipulate the Intermediaries, but, equally, the Intermediaries may try to undo this attempt. There are two dimensions along which the Sender may influence the final outcome. The first one comes from the trade-off whether to focus on attentive readers (the policy of sincerity), or on those who want to learn only mere basics (the policy of self-censorship). Since achieving both is impossible, the Sender may decide to give up on the former in order to gain on the latter. The second dimension is the level of transparency - whether to inform a single Intermediary or multiple ones. Insisting on transparency turns on competitive forces that benefit the receivers.

The received literature has observed that the outcome of communication games depends on preference differences. The main contribution of this study is to show that the outcome depends on communication capacities at least as much.

Consider the baseline scenario of perfect competition and benevolent Sender in which

The Intermediaries are equally eloquent, $R^{1}=R^{2}$,

If conditions (i)-(iii) hold then it is optimal for the Sender to opt for transparency by communicating to both Intermediaries, and announce a sincere informational policy. The Sender and the final Receiver obtain their best feasible outcome no matter what the biases of the Intermediaries are. 
If any of these conditions does not hold, the Sender's problem becomes more complicated in the sense that she may find it optimal to use selfcensorship or give up on transparency. Specifically, if (ii) and (iii) hold but (i) is replaced by $R^{1}<R^{2}$, then the more eloquent Intermediary 1 gains an advantage even if all preference parameters stay the same. He is able to tilt his message towards issues he prefers and away from the issues the Receivers and the Sender prefer. If this tilt is high enough, the Sender may even end up using self-censorship to control it. Secondly, if (i) and (iii) hold but (ii) is replaced by one-sided bias, $\beta^{1} \leq \beta^{2}<\gamma$, then the Intermediaries may coordinate to tilt the message towards issues they prefer and away from the issues the final Receivers and the Sender prefer. Again, if this tilt is high enough, the Sender may use self-censorship to control it. Of course, the benevolent Sender does not mind if the Intermediaries fail to coordinate on such a collusive equilibrium but rather continue to compete towards the best feasible communication for the Receivers. Finally, suppose that (i) and (ii) hold but (iii) is replaced by non-benevolent Sender, for example $\gamma<\alpha$. Then the Sender may choose to give up on transparency and select a single Intermediary instead, the one with closer preferences, in order to effectively collude with him, so that the message is tilted towards issues they both prefer, and away from the issues the Receiver and the other Intermediary prefer.

A more general model reveals that the policies of sincerity and selfcensorship are not the only ones that may be deployed by the Sender successfully. A policy correlating the issues together in such a way that it is impossible for the Intermediary to untangle may also be considered. Like self-censorship, this policy achieves a better communication to the less attentive public at a cost of accepting a poorer communication to the more attentive public. It turns out, however, that quite extreme assumptions on preferences are required to guarantee that such a policy is optimal.

As the final point, let us revisit a few assumptions made at the outset of this analysis. Firstly, the communication friction takes the form of a capacity constraint - zero cost of communication below capacity and infinite cost for communication exceeding capacity - rather than a smoother cost function such as linear in the "amount" of communication. This is done for the sake of simplicity. A linear cost model, although perhaps realistic in some applications, would have to contain the analysis presented below, probably without much additional insight.

Secondly, I assume that it is the initial Sender who is the Stackelberg leader. She commits to the communication policy first, then the Intermediary, who gets to know this choice, commits to her own communication policy. For example, the Sender could be a dominant, long-term player, such as a Ministry of Foreign Affairs, an important world leader, or a reputable 
think tank, while the Intermediary could be a short-termist news outlets who adjusts to whatever policy such Sender uses. One can find many examples in which alternative formulations are realistic. The Intermediaries might be long-term players, such as a well-known TV host, whose informational policy has to be accepted by small-scale or short-term Senders, such as a series of politicians, experts and personalities invited to the show. It is possible that none of the players has a commitment power, and thus assuming a simultaneous-move game in informational policies would be more appropriate.

In terms of the number of players, it is assumed that there is one Sender choosing how many Intermediaries she want to interact with. In many realistic cases, such one-to-many conversations represent only a fragment of a broader public debate, in which multiple experts of diverse interests compete with each other for the attention of the intermediaries, and so do the intermediaries for the attention of the final receivers and for access to experts. I hope that the type of analysis proposed in this paper could be used to build a more general model of market for information.

\section{Literature}

Admati, Anat R. and Paul Pfleiderer, (1986), "A Monopolistic Market for Information", Journal of Economic Theory, 39, pp. $400-438$.

Alaoui, Larbi, and Fabrizio Germano, (2014), "Time Scarcity and the Market for News", Barcelona GSE Working Paper Series, Working Paper nr 675.

Blinder, Alan S., Michael Ehrmann, Marcel Fratzscher, Jakob de Haan and David-Jan Jansen, "Central Bank Communication and Monetary Policy: A Survey of Theory and Evidence", Journal of Economic Literature, Vol. 46, No. 4 (Dec., 2008), pp. 910-945

Bodmer Report, (1985), "The public understanding of science", the Royal Society, https://royalsociety.org/topics-policy/ publications/1985/public-understanding-science/, retrieved June 11, 2019.

Crawford, Vincent P. and Joel Sobel, (1982), "Strategic Information Transmission", Econometrica, Vol. 50, No. 6, pp. 1431-1451. 
Denti, Tommaso, (2017), "Unrestricted Information Acquisition", manuscript.

Dessein, Wouter, Andrea Galeotti and Tano Santos, (2016), "Rational Inattention and Organizational Focus", American Economic Review, 106(6), June, pp. 1522-36.

El Gamal, Abbas and Young-Han Kim, (2011), Network Information Theory, Cambridge University Press.

Gaziano, Cecilie, (2016), "Knowledge Gap: History and Development", The International Encyclopedia of Media Effects, (eds) Patrick Rössler, Cynthia A. Hoffner, and Liesbet van Zoonen.

Gentzkow, Matthew and Jesse M. Shapiro, (2008), "Competition and Truth in the Market for News", Journal of Economic Perspectives, Vol 22, No 2, pp. 133-154.

Hörner, Johannes and Andrzej Skrzypacz, (2016), "Selling Information", Journal of Political Economy, vol. 124, issue 6, pp. $1515-1562$.

Kamenica, Emir, and Matthew Gentzkow, (2011), "Bayesian Persuasion", American Economic Review, 101(6), pp. 2590-2615.

Matějka, F. and A. McKay, (2015), "Rational Inattention to Discrete Choices: A New Foundation for the Multinomial Logit Model", American Economic Review, 105(1), pp. 272-98.

Matějka, F., J. Steiner and C. Stewart, (2017), "Rational Inattention Dynamics: Inertia and Delay in Decision-Making", Econometrica, 85 (2), pp. 521-553.

Milgrom, Paul, (1981), "Good News and Bad News: Representation Theorems and Applications", Bell Journal of Economics, 12, pp. 380-91.

Milgrom, Paul and John Roberts, (1986), "Relying on the Information of Interested Parties", Rand Journal of Economics, 17, pp. $18-32$.

Mullainathan, Sendhil, and Andrei Shleifer, (2005). "The Market for News", American Economic Review, 95(4), pp. 1031-53.

Nelkin, Dorothy, (1995), Selling Science, W. H. Freeman and Company, New York.

Post, Senja, (2016), "Communicating science in public controversies: Strategic considerations of the German climate scientists", Public Understanding of Science, Vol. 25(1), pp. 61-70. 
Sims, Christopher A., (2003), "Implications of Rational Inattention", Journal of Monetary Economics, 50(3), 665-690.

Sims, Christopher A., (2006), "Rational Inattention: A Research Agenda", manuscript.

Shannon, Claude (1948), "A mathematical theory of communication", Bell System Technical Journal, 27: 379-423, 623-656.

Yang, Ming (2015), "Coordination with flexible information acquisition", Journal of Economic Theory, 158, Part B (0) Symposium on Information, Coordination, and Market Frictions, pp. 721-738.

Yamamoto, Hirosuke, (1981), "Source Coding Theory for Cascade and Branching Communication Systems", IEEE Transactions on Information Theory, Vol. IT-27, No. 3, May 1981299.

\section{A Appendix: Proofs}

\section{A.1 Proof of Lemma 1}

If $R \leq \Sigma_{1} \Sigma_{2}$ then communicating $\Phi=\Sigma$ is feasible. It is also optimal since constraints (8) prevent any further decreases in distortions. Otherwise, if $R>$ $\Sigma_{1} \Sigma_{2}$, the solution must satisfy the communication frontier with equality, $R=\Phi_{1} \Phi_{2}$. Minimizing $\beta \Phi_{1}+\Phi_{2}$ subject to $R=\Phi_{1} \Phi_{2}$, (without constraints (8)) gives $(\sqrt{R / \beta}, \sqrt{\beta R})$; this is the first line of (9). The remaining two lines are cases when either constraint (8) is binding.

\section{A.2 Proof of Proposition 1}

If $R<\Sigma_{1} \Sigma_{2}$ then the Sender can lower $\Sigma_{1}$ and that will have an unambiguously positive effect on her payoff in stage one and stage two. If $S<\Sigma_{1} \Sigma_{2} \leq R$ then the Sender can lower at least one of $\Sigma_{1}$ or $\Sigma_{2}$, so that she ends up better off. Specifically, if the initial position is that the Intermediary is in the first line of (9), then lowering $\Sigma_{k}$ has no effect on $\Phi$, and so it is an improvement because of the improvement in stage one only, $\lambda>0$; if he is in the second line, then lowering $\Sigma_{1}$ has no effect on $\Phi$ and therefore it is an unambiguous improvement; likewise if the case is the third line of (9), then lowering $\Sigma_{2}$ is an improvement.

Therefore, $\Sigma_{1} \Sigma_{2}=S$. There are three regions: 
1. If $S / \sqrt{R \beta} \leq \Sigma_{1} \leq \sqrt{R / \beta}$, then the constraints (8) are not binding, and thus the Intermediary's choice is independent on the selection of $\Sigma_{1}$, by Lemma 1. In this interval, the Sender should obviously select the best sincere choice in the first stage, $\Sigma_{1}^{s}=\sqrt{S / \alpha}$, as long as this solution is in the interval. Since $\Sigma_{1}^{s} \leq \sqrt{R / \beta}$ always, the candidate solution is the following: $\Sigma_{1}^{s}$, if it is greater than the lower bound $S / \sqrt{R \beta}$, or, otherwise, this lower bound.

2. If $\Sigma_{1}<S / \sqrt{R \beta}$, then the Intermediary chooses $\Phi_{2}$ to be equal $\Sigma_{2}$, by the second line of (9). Overall, the Sender's payoff, written as a function of $\Sigma_{1}$ only, is

$$
\lambda\left(\alpha \Sigma_{1}+\frac{S}{\Sigma_{1}}\right)+(1-\lambda)\left(\alpha \Sigma_{1} \frac{R}{S}+\frac{S}{\Sigma_{1}}\right)
$$

or simply $\alpha \Sigma_{1} T / S+S / \Sigma_{1}$. Optimality in this interval is achieved at $\Sigma_{1}^{c}=S / \sqrt{T \alpha}$ and $\Sigma_{2}^{c}=\sqrt{T \alpha}$, as long as this candidate does not exceed the upper bound, $\Sigma_{1}^{c}<S / \sqrt{R \beta}$. Otherwise, the upper bound of this interval should be selected.

3. Finally, if $\sqrt{R / \beta}<\Sigma_{1}$ then the third line of (9) applies. Lowering $\Sigma_{1}$ towards the lower bound unambiguously lowers the loss of the Sender in stage one and in stage two (recall that $\alpha \geq \beta$ ). The best choice in this interval is its lower bound.

Now, we can compare the best choices in each interval to obtain the best $\Sigma_{1}$ overall. The best choice in point 1 is always better than in point 3 . Secondly, it can be easily verified that the best choice in point 1 and point 2 cannot be both equal to the $S / \sqrt{R \beta}$, one of them has to be in the interior of their respective intervals. Since the loss function is continuous at $S / \sqrt{R \beta}$, the overall solution must be either $\Sigma_{1}^{s}$ or $\Sigma_{1}^{c}$. It is the latter if the constraint in point 1 is binding, namely if $S / \sqrt{R \beta}>\Sigma_{1}^{s}$ (which is equivalent to $\beta / \alpha<$ $S / R)$. It is the former if the constraint in point 2 is binding, namely if $\Sigma_{1}^{c}>S / \sqrt{R \beta}$ (which is equivalent to $T / R<\beta / \alpha$ ). If none of them is binding (i.e. if $S / R \leq \beta / \alpha \leq T / R$; note that this interval always exists, $S<T)$, then either $\Sigma_{1}^{s}$ or $\Sigma_{1}^{c}$ may be optimal.

Which is optimal can be checked by direct comparison. Selecting $\Sigma^{s}$ implies that the loss is

$$
\lambda\left(\alpha \sqrt{\frac{S}{\alpha}}+\sqrt{\alpha S}\right)+(1-\lambda)\left(\alpha \sqrt{\frac{R}{\beta}}+\sqrt{\beta R}\right)
$$


Selecting $\Sigma^{c}$ implies that the loss is equal to $2 \sqrt{T \alpha}$. The former is lower than the latter, and the sincere point should be selected, if

$$
\frac{1}{\sqrt{\beta / \alpha}}+\sqrt{\beta / \alpha} \leq 2 A
$$

where $A=\frac{\sqrt{T}-\lambda \sqrt{S}}{(1-\lambda) \sqrt{R}}$. The left-hand side, seen as a function of $\sqrt{\beta / \alpha} \in(0,1)$, is decreasing from infinity to 2 . Number $A$ is greater than 1 . Thus, there is a threshold (which is equal to $\left(A-\sqrt{A^{2}-1}\right)^{2} \in(0,1)$ ), such that $\beta / \alpha$ greater than this threshold implies that (14) holds.

\section{A.3 Proof of Proposition 2}

The second statement follows from the first one and the fact that $B\left(R^{1}, \beta^{1}\right)$ is Intermediary 1's feasible first best. The first statement follows from $\gamma \leq \delta$ and the definition of $\delta$.

\section{A.4 Proof of Proposition 3}

Step 1: If Intermediary 1 has a strictly better capacity, $R^{1}<R^{2}$, then in any equilibrium, the final Receiver selects Intermediary 1 (this implies Corollary 1). To see this, suppose that there is an equilibrium in which Intermediary 2 is selected by the final Receiver. Clearly, Intermediary 1 can replicate the informational policy of Intermediary 2 and then lower both distortions a little, because his capacity is strictly better. This deviation of Intermediary 1 is better to all parties, including the final Receiver, who would then select this new policy, and including Intermediary 1, thus proving that the original configuration of $\left(\Phi^{1}, \Phi^{2}\right)$ is not an equilibrium.

Step 2: $\Phi^{2}=B\left(R^{2}, \gamma\right)$ and $\Phi^{1}=H(\gamma)$ forms an equilibrium. If $R^{1}<R^{2}$ then step 1 applies and the realized distortion is $H(\gamma)$. Intermediary 1 does not have incentives to deviate, by definition of $H(\gamma)$, given that Intermediary 2 offers $B\left(R^{2}, \gamma\right)$. Finally, observe that Intermediary 2 does not have any incentives to deviate either. He already offers the final Receiver the best distortion profile that he can design for the final Receiver, given rate $R^{2}$. Intermediary 2 cannot even affect the outcome, let alone improve his payoff.

Finally, if $R^{1}=R^{2}=R$, then the choices of Intermediary 1 and 2 merge into one point, $\Phi^{2}=\Phi^{1}=B(R, \gamma)$; none of these two players have any incentives to deviate.

Step 3: This equilibrium is unique. Suppose that Intermediary 2 selects some $\Phi^{2} \neq B\left(R^{2}, \gamma\right)$, and that Intermediary 1 plays a best response to that choice, $\Phi^{1}=G\left(\gamma, \Phi^{2}\right)$. However, Intermediary 2 can select a distortion 
profile that is better to the final Receiver than this $\Phi^{1}$, for example $B\left(R^{2}, \gamma\right)$ (because $\delta<\gamma$ ). Since Intermediary 2's indifference line is even steeper than the one of the final Receiver (by assumption $\gamma \leq \beta_{2}$ ) Intermediary 2 considers this distortion point better than the actually implemented $G\left(\gamma, \Phi^{2}\right)$. This profitable deviation contradicts the initial equilibrium.

\section{A.5 Proof of Proposition 4}

Consider a strategy profile $\left(\Phi^{1}, \Phi^{2}\right)$ defined in the Proposition. Intermediary 1 plays a best response to $\Phi^{2}$ by definition of $G\left(\gamma, \Phi^{2}\right)$, and this point is selected by the final Receiver. To conclude that this is an equilibrium, one has to show that Intermediary 2 does not have any incentives to deviate either. Suppose that Intermediary 2 deviates to $B\left(R^{2}, \beta^{2}\right)$, the best feasible distortion point to him, and suppose that the final Receiver selects it (which is not always assured) - it is still not better to that Intermediary 2 than $\Phi^{1}=G\left(\gamma, \Phi^{2}\right)$ obtained in the original equilibrium. To see this, note that Intermediary 2 is indifferent between $B\left(R^{2}, \beta^{2}\right)$ and $H\left(\beta^{2}\right)$, which is worse than $G\left(\gamma, \Phi^{2}\right)$.

To verify point 1 of the Proposition, consider a feasible choice of Intermediary 2, such that $\Phi^{2} \notin \Delta$, and a corresponding choice of Intermediary $1, G\left(\gamma, \Phi^{2}\right)$. It turns out that in this configuration, Intermediary 2 has incentives to deviate. Namely, since $\delta<\beta^{2}$, profile $G\left(\gamma, \Phi^{2}\right)$ is worse to Intermediary 2 than $H\left(\beta^{2}\right)$, which in turn is indifferent to his best feasible outcome, $B\left(R^{2}, \beta^{2}\right)$. That is, Intermediary 2 would prefer to deviate to $B\left(R^{2}, \beta^{2}\right)$, if he could only make sure that the final Receiver selected that point. But since this is the two-sided bias case, $\beta^{2}<\gamma$, the final Receiver prefers $B\left(R^{2}, \beta^{2}\right)$ to $G\left(\gamma, \Phi^{2}\right)$, so she would select it.

To verify point 2 , observe that Intermediary 1 's best response to such $\Phi^{2}$ is $G\left(\gamma, \Phi^{2}\right)=B\left(R^{1}, \beta^{1}\right)$. However, since $\beta_{2} \leq \delta$, no distortion profile feasible to Intermediary 2 is better to him than the final outcome generated by Intermediary 1 . 